\title{
Modeling Residence Time Distribution (RTD) Behavior in a Packed-Bed Electrochemical Reactor (PBER)
}

\author{
Sananth H. Menon $\mathbb{D D}^{1}{ }^{1}$ G. Madhu, ${ }^{2}$ and Jojo Mathew ${ }^{1}$ \\ ${ }^{1}$ Ammonium Perchlorate Experimental Plant, Vikram Sarabhai Space Centre, ISRO, Aluva, India \\ ${ }^{2}$ School of Engineering, Cochin University of Science \& Technology, Kochi, India \\ Correspondence should be addressed to Sananth H. Menon; sananthisro@gmail.com
}

Received 28 July 2018; Revised 15 December 2018; Accepted 19 January 2019; Published 26 February 2019

Academic Editor: Deepak Kunzru

Copyright $\odot 2019$ Sananth H. Menon et al. This is an open access article distributed under the Creative Commons Attribution License, which permits unrestricted use, distribution, and reproduction in any medium, provided the original work is properly cited.

This paper focuses on understanding the electrolyte flow characteristics in a typical packed-bed electrochemical reactor using Residence Time Distribution (RTD) studies. RTD behavior was critically analyzed using tracer studies at various flow rates, initially under nonelectrolyzing conditions. Validation of these results using available theoretical models was carried out. Significant disparity in RTD curves under electrolyzing conditions was examined and details are recorded. Finally, a suitable mathematical model (Modified Dispersed Plug Flow Model (MDPFM)) was developed for validating these results under electrolyzing conditions.

\section{Introduction}

It is known that a packed-bed electrochemical reactor having particulate electrodes can provide a relatively large electrode surface when compared with a conventional flat-electrode configuration. Consequently, this packed-bed electrolyzer will be remarkably useful when dealing with low reactant concentrations or slow reactions [1-4]. They also find a better alternate for large-scale manufacturing of basic chemicals and intermediates as well as for the removal of harmful or toxic chemicals from gas or liquid streams [5].

Flow behavior of electrolyte through these reactors via RTD studies has been one of the key components in understanding its vessel hydrodynamics. In an experimental study of residence-time distribution, flow elements are tagged by a tracer (colored, radioactive, etc.) and the variation of tracer concentration in the exit stream with time is measured. The injection of tracer into the flow stream is frequently done in such a manner that it can be well approximated by a delta function or a thin finite width pulse. The tracer concentration distribution at the exit (called also the tracer output signal) has a characteristic shape depending upon the relative strength of dispersion and on the location of tracer injection and detection.
Developing a suitable theoretical model justifying RTD behavior has been an onus among the engineers for quite a long period of time. Not surprisingly, various studies were reported exhibiting peculiar flow behaviors in variety of systems. Saravanathamizhan et al. [6] provided a threeparameter model to describe the electrolyte flow in continuous stirred tank electrochemical reactor (CSTER) consisting bypass, active, and dead zones with exchange flow between active and dead zones. The authors validated the model for the effluent color removal inside a typical CSTER. Atmakidis and Kenig [7] conducted a numerical analysis of dispersion in packed beds and developed an RTD model using CFD modeling. Benhabiles et al. [8] conducted the experimental study of photo catalytic degradation of an aqueous solution of linuron in a tubular type reactor and used RTD data for investigating the malfunction of the photo reactor. Martin [9] showed that ETIS (extension to tanks in series) model in tandem with the reactor network structure is a versatile method of describing the characteristics of a small but diverse group of reactors.

Earlier studies had shown that conventional models [10] like open dispersion models, small dispersion models, and tanks in series models can explain with lot of clarity the behavior of electrolyte inside a typical packed-bed reactor 
under nonelectrolyzing conditions. However, not many studies were reported in the literature regarding the applicability of a suitable model in a packed bed reactor operating under electrolyzing conditions. Many of these models fail to explain the recirculation flow expected inside such reactor due to obvious gas evolution around the particulate electrodes. In the initial phase of present study, we made a detailed interpretation of electrolyte flow inside a packed-bed electrochemical bed reactor under nonelectrolyzing conditions using an experimental RTD analysis. Applicability of available theoretical models was also carried out to strengthen the experimental findings. At a later part of the study, similar RTD studies were repeated at analogous flow conditions but operated under electrolyzing environment, for getting comparative flow behaviors with and without electrolyzing environment. A Modified Dispersed Plug Flow Model (MDPFM) was developed to validate the variation in such flow circumstances.

\section{Experimental Details}

Experiments were carried out in the packed-bed electrochemical reactor schematically presented in [1]. The cell was of cylindrical geometry and was made of high-density polyethylene (HDPE); the overall dimensions were $0.178 \mathrm{~m}$ (ID) $\times 0.30 \mathrm{~m}(\mathrm{H})$. Particle size distribution of these particles obtained using sieve analysis and its image analysis using zoom stereoscopic microscope are mentioned in Tables 1 and 2, respectively. Shape-scrapped lead dioxide particles (about $3.5 \mathrm{Kg}$ ) were thoroughly cleaned using DI water and closely packed in the electrolyzer up to $5 \mathrm{~cm}$ height. Perforated polypropylene supports (which also serves as distributor) with nylon mesh filter were used at both ends for ensuring rigid and leak proof packing. For carrying out RTD studies, methylene blue having a concentration of $40 / 80 \mathrm{ppm}$ was selected as tracer. Tracer was injected to cell closer to its inlet at about $2 \mathrm{~cm}$ from HDPE body through a Tee provided at the feed bottom. Flow medium used was water for conducting studies in the nonelectrolytic mode. On the contrary, sodium chlorate solution having $5 \mathrm{Kg} / \mathrm{m}^{3}$ concentrations was chosen for studies in the electrolytic mode. Flow rates of the medium varied from $3.33 \times 10^{-5} \mathrm{~m}^{3} / \mathrm{sec}$ to $1.33 \times 10^{-4} \mathrm{~m}^{3} / \mathrm{sec}$ for understanding the variation in RTD behavior. A DC current of $15 \mathrm{~A}$ was fed into the electrolyzer (under electrolytic mode) using as Rectifier having $200 \mathrm{~A}$, $60 \mathrm{~V}$ specification. A double-beam UV spectrometer was used for estimating the transient variation in concentration of tracer in effluent, indirectly by measuring the color intensity. Figures 1 and 2 show the schematic experimental setup.

\section{Modeling RTD Behavior}

3.1. Open Dispersion Model. This model predicts that electrolyte flow in the PBER is undisturbed at the inlet and outlet. The fundamental mathematical form is

$$
\frac{\partial C}{\partial \theta}=\left(\frac{D}{u \cdot L}\right) \frac{\partial^{2} C}{\partial z^{2}}-\frac{\partial C}{\partial z}
$$

TABLE 1

\begin{tabular}{lcc}
\hline & Particle size distribution & \\
Size range & Weight (\%) \\
\hline$>710 \mu$ & 87.53 \\
$500-710 \mu$ & 9.46 \\
$355-500 \mu$ & 2.28 \\
$300-355 \mu$ & 0.27 \\
$<300 \mu$ & 0.46 \\
\hline
\end{tabular}

TABle 2

\begin{tabular}{lccc}
\hline \multirow{2}{*}{ Sl no. } & Sample ref. & \multicolumn{2}{c}{ Size in microns } \\
& & Min & Max \\
\hline 1 & Fine $\mathrm{PbO}_{2}-1$ & 721.8 & 1756.6 \\
2 & Fine $\mathrm{PbO}_{2}-2$ & 485 & 837.4 \\
3 & Medium $\mathrm{PbO}_{2}-2$ & 1029.3 & 1245.4 \\
4 & Medium $\mathrm{PbO}_{2}-2$ & 1182.8 & 3285.7 \\
5 & Coarse $\mathrm{PbO}_{2}-1$ & 767.7 & 3341.9 \\
6 & Coarse $\mathrm{PbO}_{2}-2$ & 482.9 & 9439.4 \\
\hline
\end{tabular}

Boundary conditions from [11] are as follows.

For open system, at entrance, $F_{\mathrm{T}}\left(0^{-}, t\right)=F_{\mathrm{T}}\left(0^{+}, t\right)$. That is, $\quad-D\left(\partial C_{\mathrm{T}} / \partial z\right)_{z=0}^{-}+U C_{\mathrm{T}}\left(0^{-}, t\right)=-D\left(\partial C_{\mathrm{T}} / \partial z\right)_{z=0}^{+}+U C_{\mathrm{T}}$ $\left(0^{+}, t\right)$ or $C_{T}\left(0^{-}, t\right)=C_{T}\left(0^{+}, t\right)$.

At the exit,

$$
\begin{aligned}
C_{\mathrm{T}}\left(0^{-}, t\right) & =C_{\mathrm{T}}\left(0^{+}, t\right), \\
-D\left(\frac{\partial C_{\mathrm{T}}}{\partial z}\right)_{z=L}^{-}+U C_{\mathrm{T}}\left(L^{-}, t\right) & =-D\left(\frac{\partial C_{\mathrm{T}}}{\partial z}\right)_{z=L}^{+}+U C_{\mathrm{T}}\left(L^{+}, t\right) .
\end{aligned}
$$

Analytical solution of (1) from [10] is as follows:

$$
E(\theta)=\frac{C}{C_{d}}=\left(\frac{1}{\sqrt{4 \pi(D / u \cdot L)}}\right) \cdot \exp \left[\frac{-(1-\theta)^{2} u \cdot L}{4 \theta \cdot D}\right] \text {. }
$$

3.2. Model Predicting Small Extent of Dispersion (Small Dispersion). For small extent of dispersion, the spreading tracer curve does not change its shape as it passes the measuring point. This yields a symmetric curve and analytical solution is as follows:

$$
E(\theta)=\frac{C}{C_{\mathrm{d}}}=\left(\frac{1}{\sqrt{4 \pi(D / u \cdot L)}}\right) \cdot \exp \left[\frac{-(1-\theta)^{2} u \cdot L}{4 \cdot D}\right] .
$$

3.3. Tanks in Series Model. This model predicts that electrolyte flow in PBER is discretised into equal sized hypothetical CSTR's. The number of tanks in series $n_{\mathrm{T}}$ describes the dispersion with $n_{\mathrm{T}}=1$ representing infinite dispersion and being equivalent to $\mathrm{Pe}=0$. Analytical solution which is also the definition of Erlang distribution is as follows: 


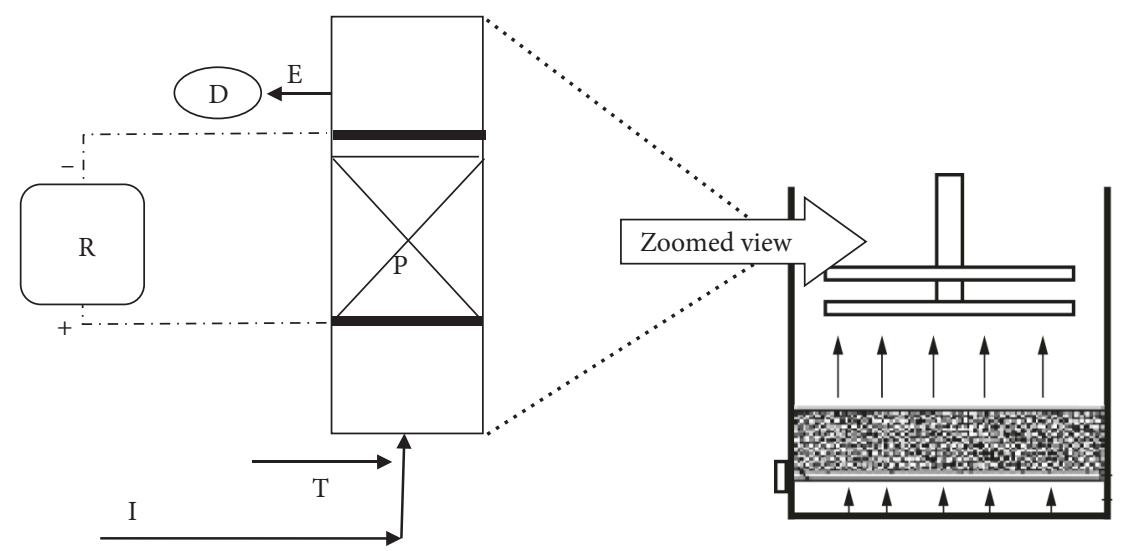

Figure 1: Schematic sketch of experimental setup. P: PBER; T: tracer in; I: influent stream; E: effluent stream; R: rectifier; D: spectrophotometer.

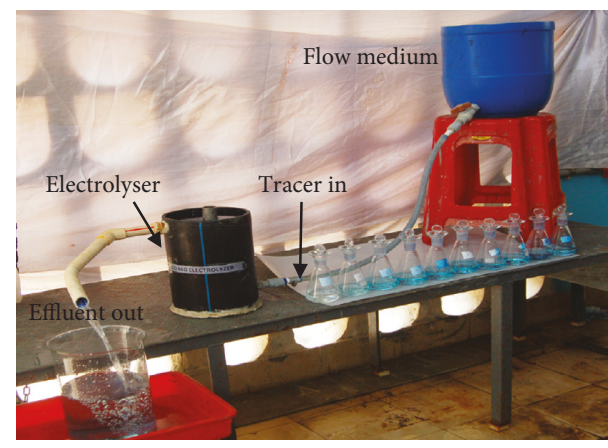

FIgURE 2: Experimental setup.

$$
E(\theta)=\frac{C}{C_{\mathrm{d}}}=\frac{n_{\mathrm{T}}^{n_{\mathrm{T}}}}{\left(n_{\mathrm{T}}-1\right) ! \theta_{\mathrm{T}}^{\left(n_{\mathrm{T}}-1\right)} e^{-n} T^{\theta}} .
$$

\section{Results and Discussion}

4.1. RTD Curves at Various Flow Rates. RTD behavior of PBER under various electrolyte flows is depicted in Figures 3(a)-3(d). Table 3 shows the calculated $1^{\text {st }}$ and $2^{\text {nd }}$ moment about mean. Reasonably good flow was observed through the reactor at $3.33 \times 10^{-5} \mathrm{~m}^{3} / \mathrm{sec}$, as the mean time interval falls at the right place. As the flow rate is increased, the curve shifts towards the left indicating the presence of early time mean. This observation along with long tail indicates the presence of stagnant backwaters. This can be ascertained by comparing the space time under each flow rate with the observed mean from graph [10]. Table 4 indicates that percentage difference predominantly increases at higher flows substantiating the presence of stagnant regions.

4.2. Modeling RTD Behavior. Let $Q_{\mathrm{R}}$ be the flow of electrolyte through the bed and $V_{\mathrm{R}}$ be the volume of PBER. Then tank residence time $T_{\mathrm{R}}=V_{\mathrm{R}} / \mathrm{Q}_{\mathrm{R}}$. All time domains were converted to dimensionless $\theta$ mode, where $\theta=t / T_{\mathrm{R}}$. From the respective exit age distribution curves, $\sigma^{2}$ and $t_{\text {mean }}$ are estimated.
Then,

$$
\sigma_{\theta}^{2}=\frac{\sigma^{2}}{t_{\text {mean }}^{2}} .
$$

Peclet no. can be found out using the following equation:

$$
\sigma_{\theta}^{2}=2 \times \mathrm{D} /(u \cdot L)-2 \times(D /(u \cdot L))^{2} \times\left(1-e^{(-u \cdot L / D)}\right) .
$$

The aforementioned parameters were inserted in respective modeling equations mentioned in equations (2) and (3) to get the predicted $E_{\theta}$ values using the Open dispersion model and model predicting small extent of dispersion, respectively. For tanks in series model, following equations were used:

$$
\begin{aligned}
& \operatorname{Pe}=2\left(n_{\mathrm{T}}-1\right), \\
& E_{\theta}=\frac{n_{\mathrm{T}}^{n_{\mathrm{T}}}}{\left(n_{\mathrm{T}}-1\right) ! \cdot \theta_{\mathrm{T}}^{n-1} \cdot e^{-n_{\mathrm{T}} \cdot \theta}},
\end{aligned}
$$

where $n_{\mathrm{T}}$ represents the number of tanks in series.

Using these parameters and respective model equations, $E_{\theta}$ was analytically determined under various flow rates. Figure 4 shows the graphical representation of these models.

From Figures $4(\mathrm{a})-4(\mathrm{~d})$, it is quite explicit that RTD behavior of PBER can be well approximated by the Open dispersion model and model predicting small extent of dispersion. Though Tank in Series model could predict at higher flow rates, significant disparities could be seen at lower flows. In order to estimate the extent of dispersion, Vessel Dispersion number $(D / u \cdot L)$ was determined from equation (7), and the same was plotted under various flow rates in Figure 5. Table 4 shows the calculated values of $D /$ $u \cdot L$. It is observed that $D / u \cdot L$ values increases till the flow reaches 6 LPM and beyond which it decreases. It shows that axial dispersion coefficients competes for their prominence with obvious backmixing owing to recirculation flows observed in high flow rates. This is the probable reason for decrease in $D / u \cdot L$ values under high flow rates.

4.3. RTD Curves under Electrolyzing Conditions. Figure 6 shows RTD behavior under electrolyzing conditions at 


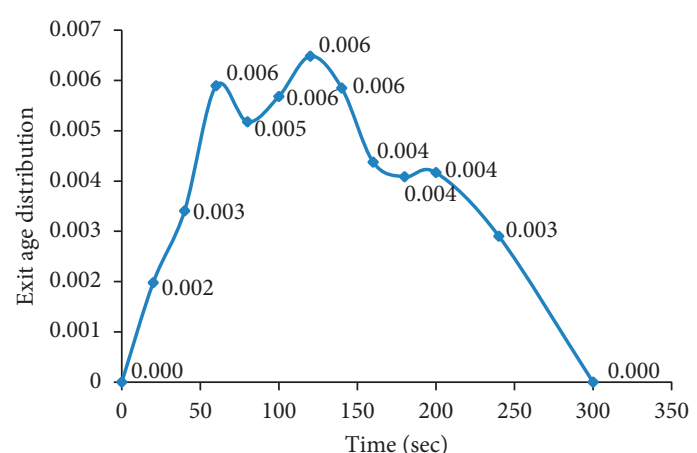

(a)

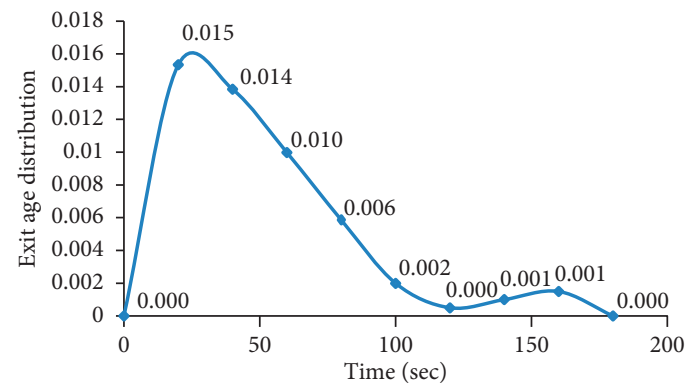

(c)

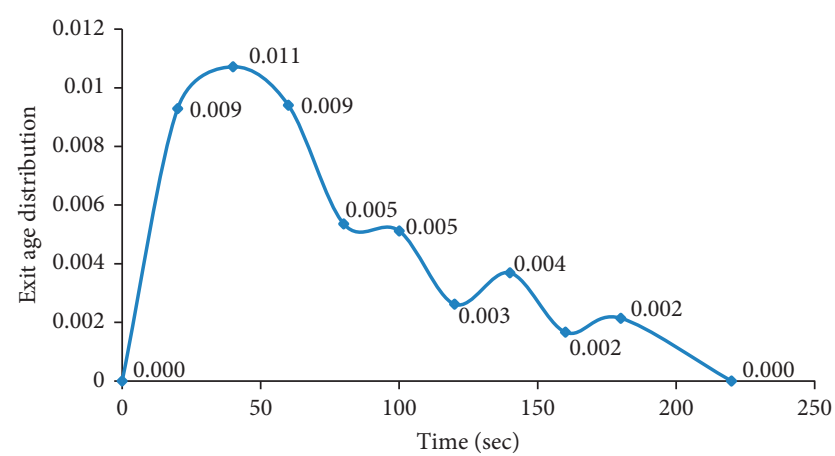

(b)

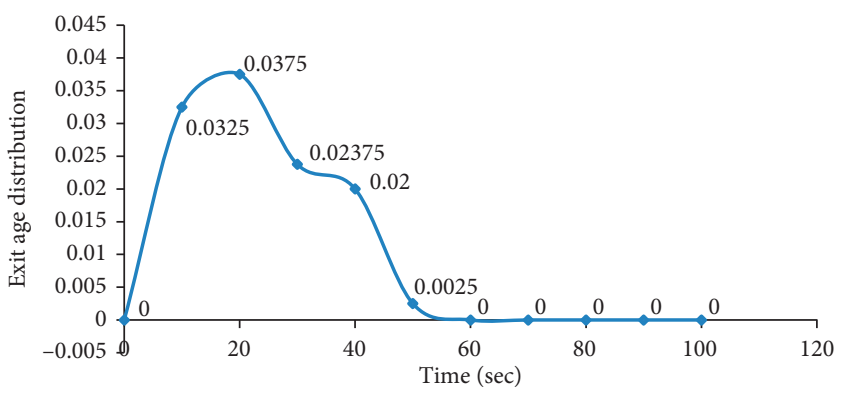

(d)

Figure 3: RTD behavior under various electrolyte flow rates. (a) At $3.33 \times 10^{-5} \mathrm{~m}^{3} / \mathrm{sec}$. (b) At $6.67 \times 10^{-5} \mathrm{~m}^{3} / \mathrm{sec}$. (c) At $1 \times 10^{-4} \mathrm{~m}^{3} / \mathrm{sec}$. (d) At $1.33 \times 10^{-4} \mathrm{~m}^{3} / \mathrm{sec}$.

TABLE 3

\begin{tabular}{lccc}
\hline Sl no. & Flow rate $\left(\mathrm{m}^{3} / \mathrm{sec}\right)$ & $1^{\text {st }}$ moment $(\mathrm{sec}) t_{\text {mean }}=\int_{0}^{\infty} t \cdot E(t) d t$ & $2^{\text {nd }}$ moment about mean $\left(\sec ^{2}\right) \sigma^{2}=\int_{0}^{\infty}\left(t-t_{\text {mean }}\right)^{2} E(t) d t$ \\
\hline 1 & $3.33 \times 10^{-5}$ & 141.48 & 3257 \\
2 & $6.67 \times 10^{-5}$ & 65.05 & 2339.5 \\
3 & $1 \times 10^{-4}$ & 41.32 & 1109 \\
4 & $1.33 \times 10^{-4}$ & 22.35 & 153.33 \\
\hline
\end{tabular}

TABLE 4

\begin{tabular}{lcccc}
\hline Sl no. & Flow rate $\left(\mathrm{m}^{3} / \mathrm{sec}\right)$ & Space time $(\tau)(\mathrm{sec})$ & $t_{\text {mean }}$ (observed from Figure 3$)(\mathrm{sec})$ & Difference $\left(\tau-t_{\text {mean }}\right)(\%)$ \\
\hline 1 & $3.33 \times 10^{-5}$ & 150 & 141.48 & 6 \\
2 & $6.67 \times 10^{-5}$ & 75 & 65.05 & 10.64 \\
3 & $1 \times 10^{-4}$ & 50 & 41.32 & 21 \\
4 & $1.33 \times 10^{-4}$ & 37.5 & 22.35 & 67.78 \\
\hline
\end{tabular}

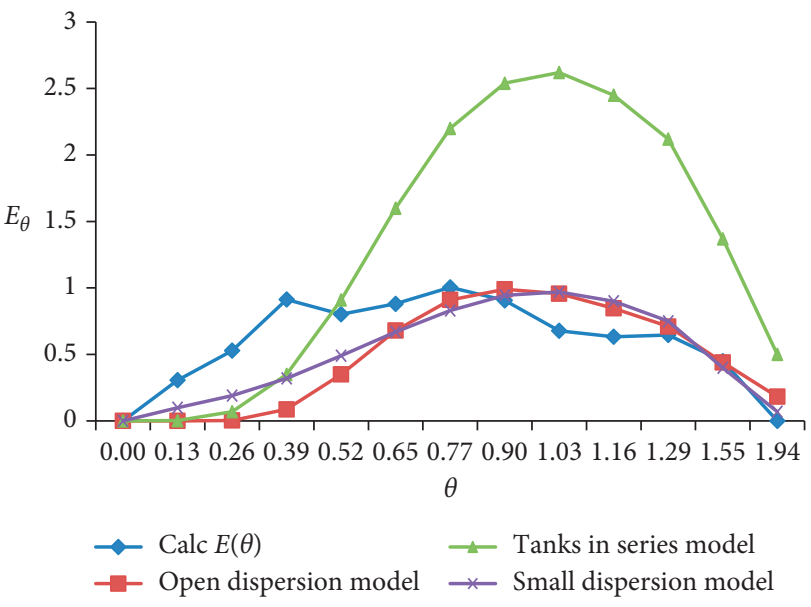

(a)

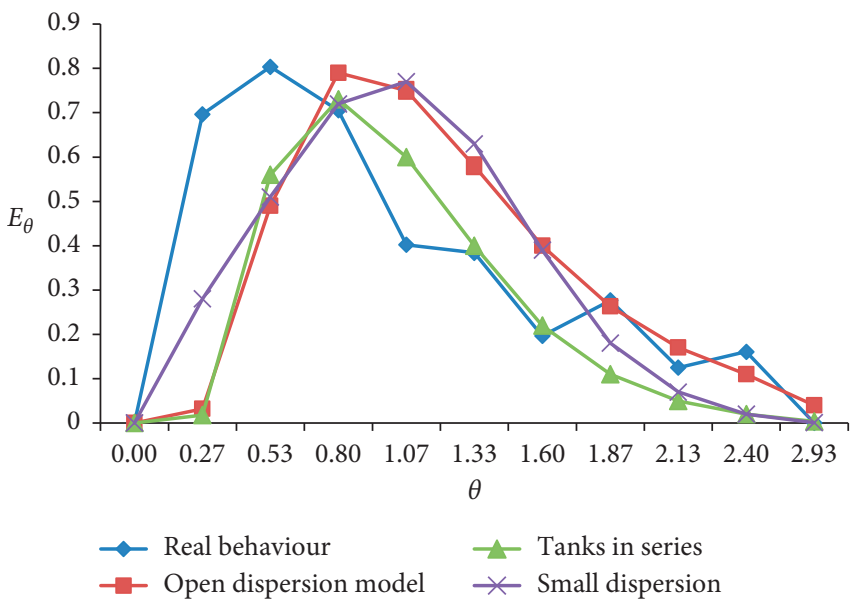

(b)

Figure 4: Continued. 


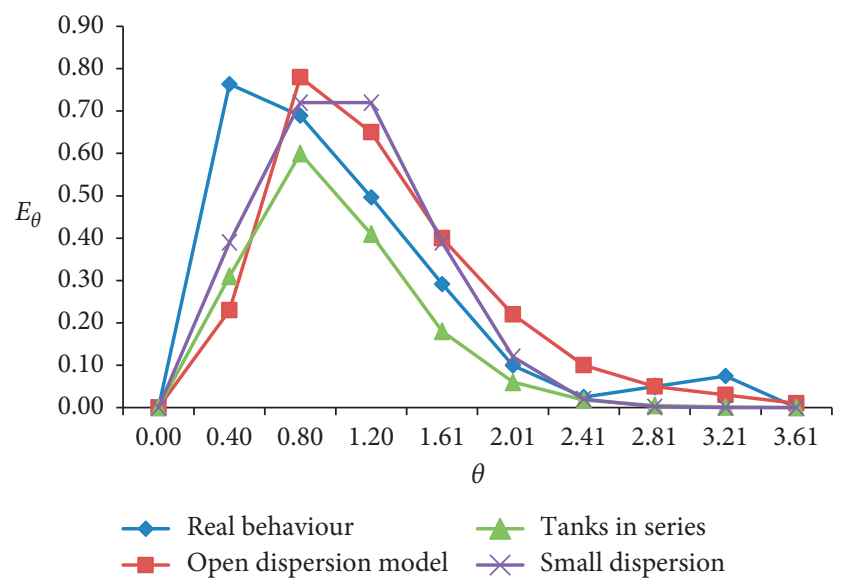

(c)

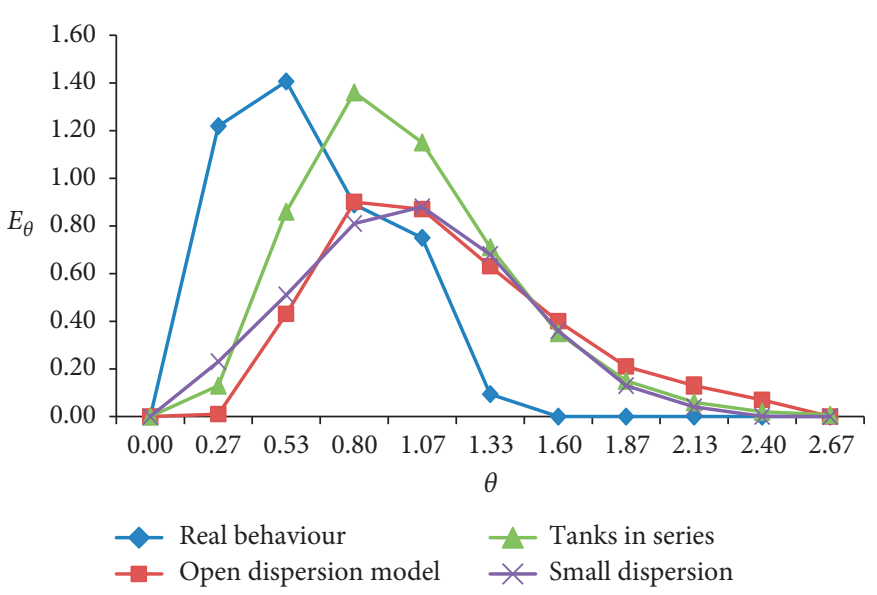

(d)

Figure 4: Modeling RTD behavior under various electrolyte flow rates. (a) At $3.33 \times 10^{-5} \mathrm{~m}^{3} / \mathrm{sec}$. (b) At $6.67 \times 10^{-5} \mathrm{~m}^{3} / \mathrm{sec}$. (c) At $1 \times 10^{-4} \mathrm{~m}^{3} / \mathrm{sec}$. (d) At $1.33 \times 10^{-4} \mathrm{~m}^{3} / \mathrm{sec}$.

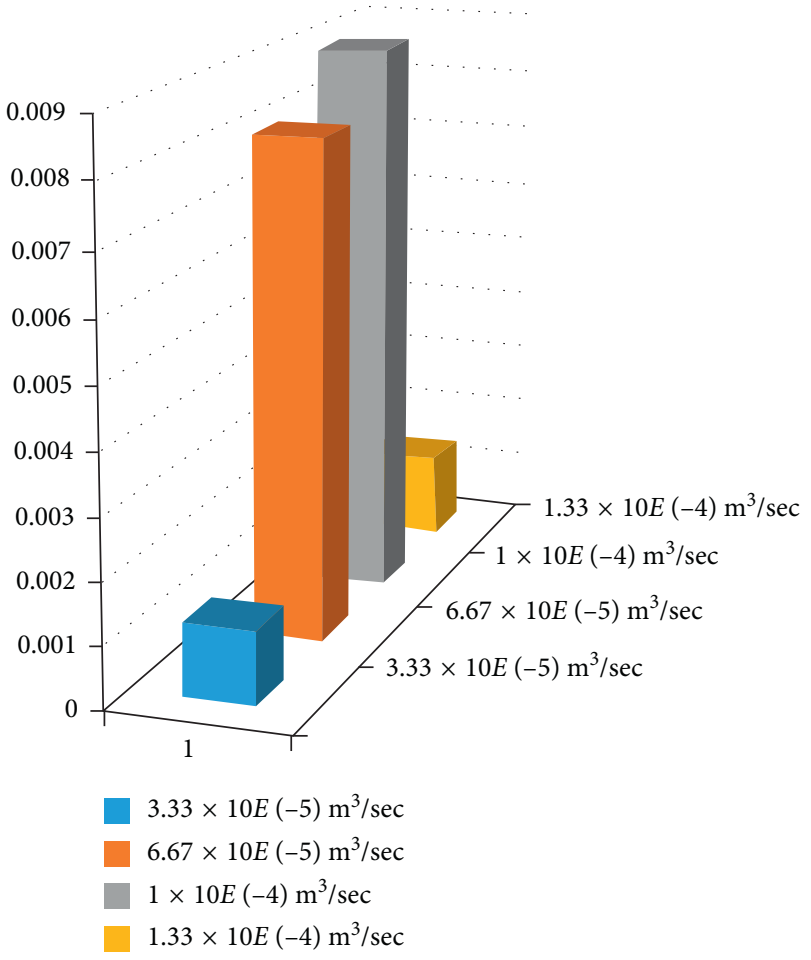

Figure 5: Plot of $D / u \cdot L$ for various flow rates.

various flow rates. Table 5 represents the values of vessel dispersion number for various flow rates. Table 6 shows the calculated $1^{\text {st }}$ and $2^{\text {nd }}$ moment about mean. In general, an axial dispersion effect gets diminished under the electrolyzing mode at intermediate flow rates. This is primarily because nonideal axial flow currents gets disturbed by the back flow currents generated by gases which are inevitably produced at the electrode surface under electrolyzing conditions. Recirculation, channeling, and short circuit flows observed under certain flow conditions got totally eliminated under electrolyzing mode which may obviously be due to adequate backmixing of electrolyte between the particles, contributed by the gases generated around these electrode particles.

4.4. Modified Dispersed Plug Flow Model (MDPFM) under Electrolyzing Conditions. Refer to Appendix for detailed analytical treatment.

This model assumes that a fraction of recirculation flow $(\alpha)$ generated due to gaseous evolution around the electrodes flows back to the conventional platform of dispersed plug flow. It is pictorially denoted in Figure 7.

Exit age distribution $E_{\theta}$ as per dispersed plug flow model $=1 / \sqrt{\left(4 \pi \mathrm{Pe}^{-1}\right) \times e^{-[(1-\theta) 2 \mathrm{Pe} / 4]}}$.

Thus, as per the above model,

$$
\begin{aligned}
& \frac{C_{1}^{\prime}(t)}{C_{0}}=\frac{1}{\sqrt{\left(4 \pi \mathrm{Pe}^{-1}\right)}} \times e^{-\left[\left(1-t / T_{\mathrm{R}}\right) 2 \mathrm{Pe} / 4\right]}, \\
& C_{1}^{\prime}(t)=\frac{C_{0}}{\sqrt{\left(4 \pi \mathrm{Pe}^{-1}\right)}} \times e^{-\left[\left(1-t / T_{\mathrm{R}}\right) 2 \mathrm{Pe} / 4\right]}
\end{aligned}
$$

4.4.1. Taking Material Balances.

$$
Q+\alpha Q=Q(1+\alpha) .
$$

At junction point $\mathrm{M}$,

$$
Q(1+\alpha) C_{1}^{\prime}=Q \cdot C_{1}+\alpha q \cdot \delta(t=0) .
$$

Taking Laplace transform across (11),

$$
Q \cdot C_{1}(s)+\alpha q=Q(1+\alpha) C_{1}^{\prime}(s) .
$$

We know

$$
C 1^{\prime}(s)=\int_{0}^{\infty} e^{-s t} C_{1}(t) d t
$$

Using standard results from integration, 


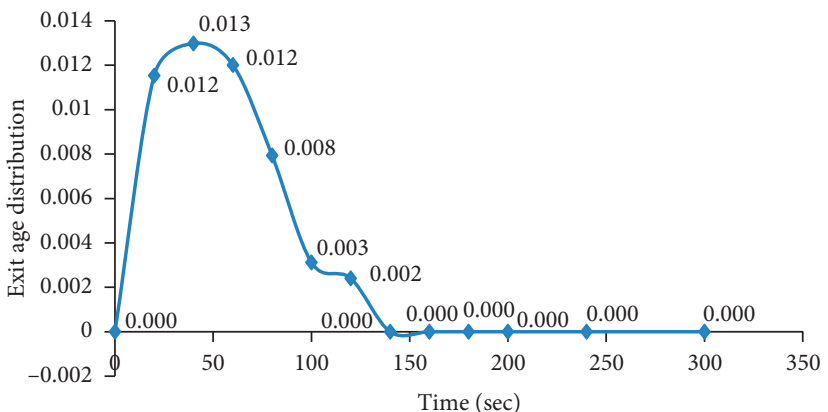

(a)

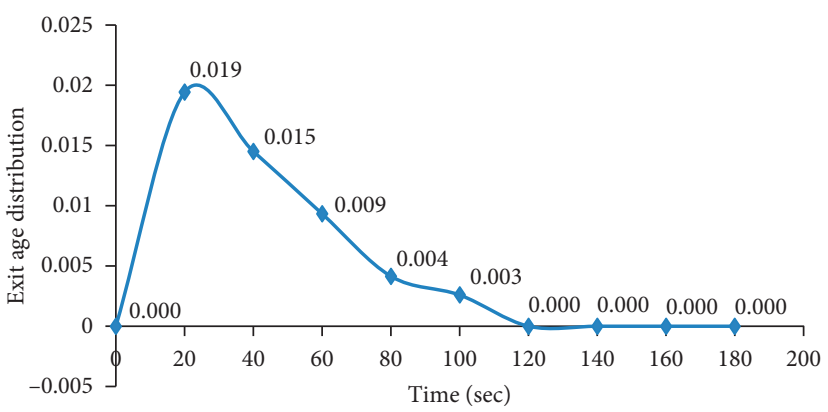

(c)

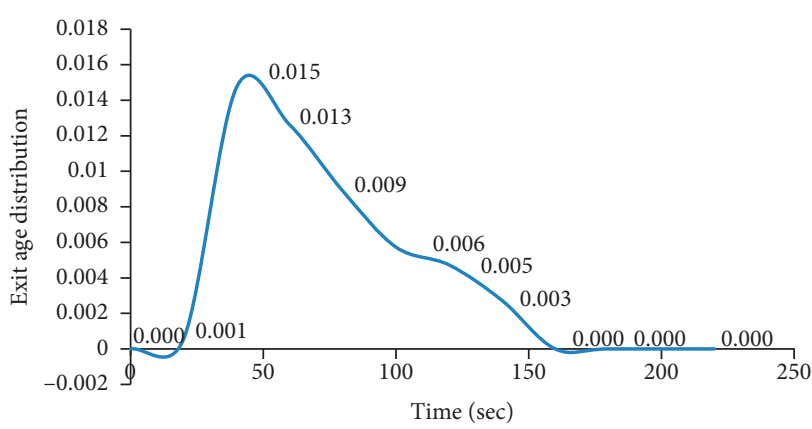

(b)

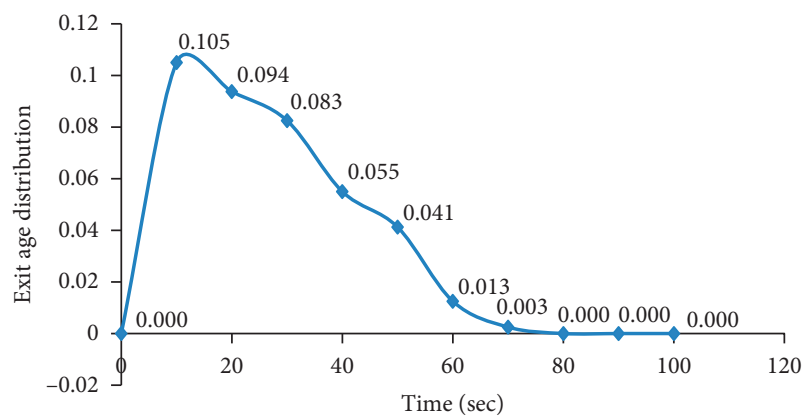

(d)

Figure 6: RTD behavior under various electrolyte flow rates. (a) At $3.33 \times 10^{-5} \mathrm{~m}^{3} / \mathrm{sec}$. (b) At $6.67 \times 10^{-5} \mathrm{~m}^{3} / \mathrm{sec}$. (c) At $1 \times 10^{-4} \mathrm{~m}^{3} / \mathrm{sec}$. (d) At $1.33 \times 10^{-4} \mathrm{~m}^{3} / \mathrm{sec}$.

TABle 5

\begin{tabular}{lccc}
\hline Sl no. & $\begin{array}{c}\text { Flow rate } \\
\left(\mathrm{m}^{3} / \mathrm{sec}\right)\end{array}$ & $\begin{array}{c}\sigma^{2} \theta \\
(\text { from graph) }\end{array}$ & $\begin{array}{c}D / u \cdot L \\
\text { (from equation }(7))\end{array}$ \\
\hline 1 & $3.33 \times 10^{-5}$ & 0.0024 & 0.0012 \\
2 & $6.67 \times 10^{-5}$ & 0.016 & 0.0081 \\
3 & $1 \times 10^{-4}$ & 0.0178 & 0.009 \\
4 & $1.33 \times 10^{-4}$ & 0.0027 & 0.00136 \\
\hline
\end{tabular}

TABle 6

\begin{tabular}{cccc}
\hline Sl no. & $\begin{array}{c}\text { Flow rate } \\
\left(\mathrm{m}^{3} / \mathrm{sec}\right)\end{array}$ & $\begin{array}{c}1^{\text {st }} \text { moment }(\mathrm{sec}) \\
t_{\text {mean }}=\int_{0}^{\infty} t \cdot E(t) d t\end{array}$ & $\begin{array}{c}2^{\text {nd }} \text { moment about } \\
\text { mean }\left(\mathrm{sec}^{2}\right) \sigma^{2}= \\
\left(t-t_{\text {mean }}\right)^{2} E(t) d t\end{array}$ \\
\hline 1 & $3.33 \times 10^{-5}$ & 64.13 & 775.71 \\
2 & $6.67 \times 10^{-5}$ & 59.2 & 741 \\
3 & $1 \times 10^{-4}$ & 42.38 & 544 \\
4 & $1.33 \times 10^{-4}$ & 27 & 217 \\
\hline
\end{tabular}

$$
\begin{aligned}
\mathrm{C}_{1}(\mathbf{s})= & (1+\alpha) \frac{C_{0}}{\sqrt{\left(4 \pi \mathrm{Pe}^{-1}\right)}} \times T_{\mathrm{R}} \sqrt{\pi \mathrm{Pe}^{-1}} \\
& \times \operatorname{erfc}\left(\sqrt{\mathrm{Pe}^{-1}} \cdot T_{R}\left(s+\left(0.5 \times \frac{\mathrm{Pe}}{T_{R}}\right)\right)\right) \\
& \times e^{\left[(1 / \mathrm{Pe}) \cdot T_{\mathrm{R}} 2\left(s 2+s \cdot \mathrm{Pe} / T_{\mathrm{R}}\right)\right]}-\frac{\alpha q}{\mathrm{Q}}
\end{aligned}
$$

4.4.2. Finding $C_{1}(t)$ by Taking Inverse Laplace Transform of $C_{1}(s)$. Comparing from the standard form of results for inverse Laplace transform for product form of exponential and error function,

$$
\begin{gathered}
L^{-1}\left[e^{k 2 \cdot s 2} \times \operatorname{erfc}(k s)\right] \quad k>0=\frac{1}{(k \sqrt{\pi})} \cdot e^{(-(t 2 / 4 k 2))}, \\
C_{1}(t)=(1+\alpha) \cdot \frac{C_{0}}{\sqrt{\left(4 \pi \mathrm{Pe}^{-1}\right)}} \cdot T_{\mathrm{R}} \sqrt{\left(\pi \cdot \mathrm{Pe}^{-1}\right)} \\
\cdot \frac{1}{(k \cdot \sqrt{\pi})} \cdot e^{-\mathrm{Pe}} \cdot e^{(-(t 2 / 4 k 2))} \cdot e^{\left(-\left(\mathrm{Pe} / 2 T_{\mathrm{R}}\right)\right) t}-\frac{\alpha q}{\mathrm{Q}} .
\end{gathered}
$$

Rearranging (15),

$$
C_{1}(t)=\left[0.5(1+\alpha) C_{0} \cdot \frac{T_{\mathrm{R}}}{k \sqrt{\pi}} \cdot e^{(-0.5 \mathrm{Pe}(\theta+\theta 2 / 2))}-\frac{\alpha q}{\mathrm{Q}}\right]
$$

Further simplifying and putting residence time distribution function as $E(\theta)=C_{1}(t) / C_{0}$ (from equation (A.13)),

$$
E(\theta)=(1+\alpha) \cdot \frac{e^{-\mathrm{Pe}}}{\left(2 \cdot \sqrt{ } \pi \mathrm{Pe}^{-1}\right)} \times e^{(-0.5 \mathrm{Pe}(\theta+\theta 2 / 2))}-\frac{\alpha q}{Q C_{0}}
$$

4.5. Validation of MDPFM. By putting $\alpha=0.5$, equation (17) was used to predict the values of $E(\theta)$ under various flow rates. Figure 8 represents the comparison with the actual behavior. 


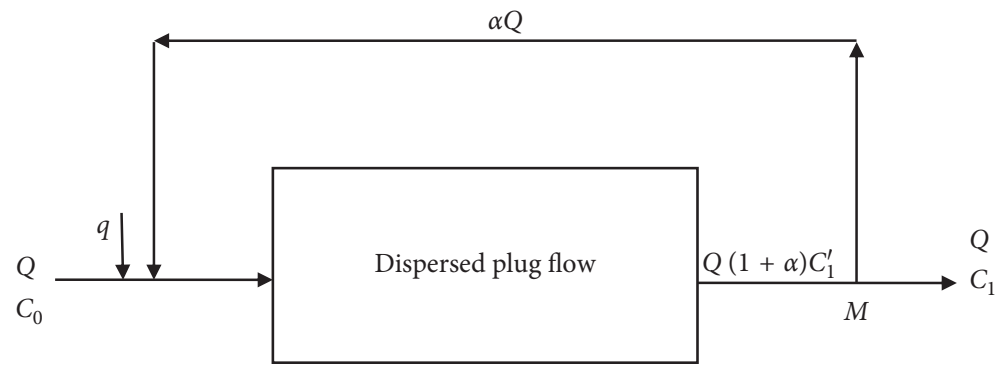

Figure 7: MDPM conceptual block diagram.

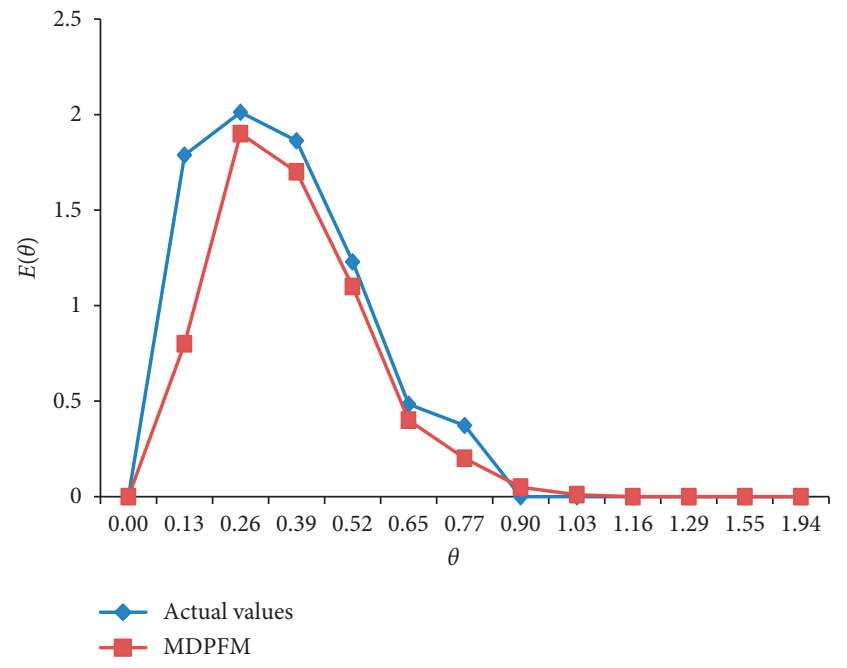

(a)

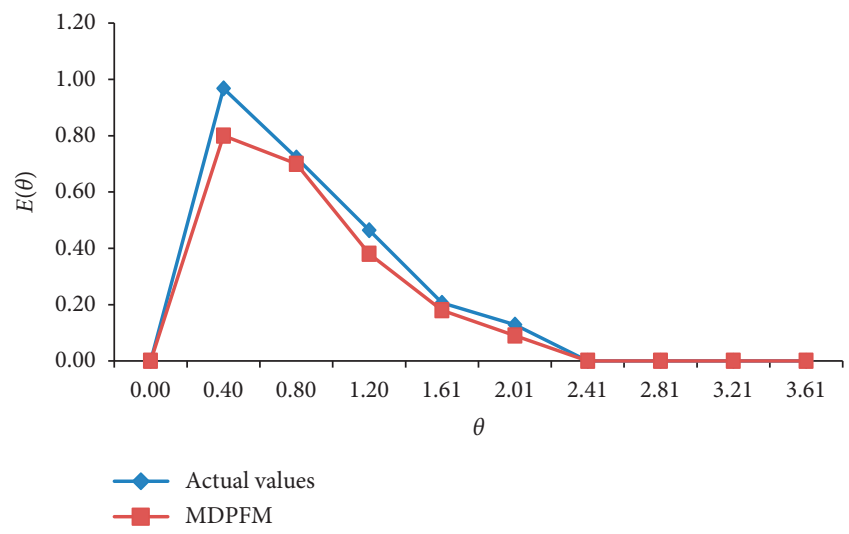

(c)

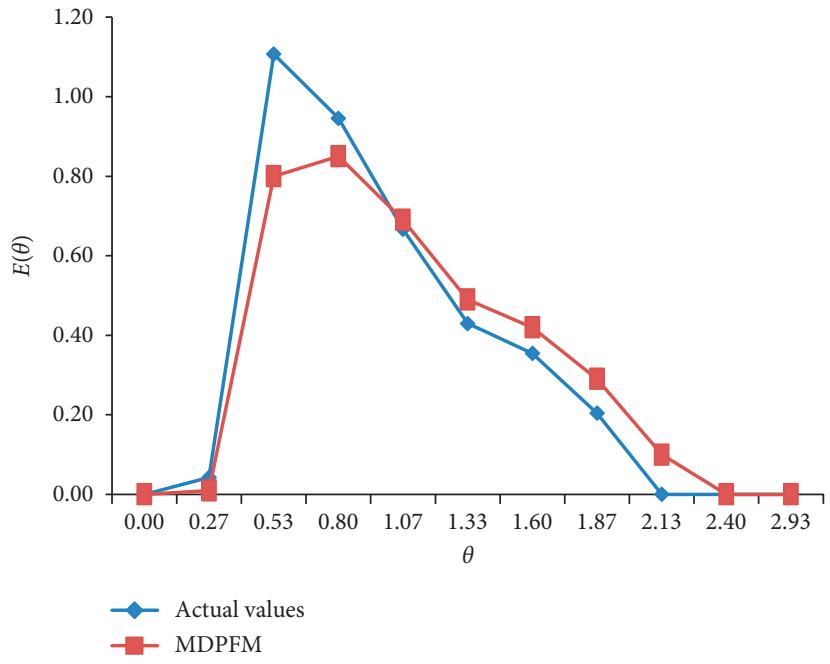

(b)

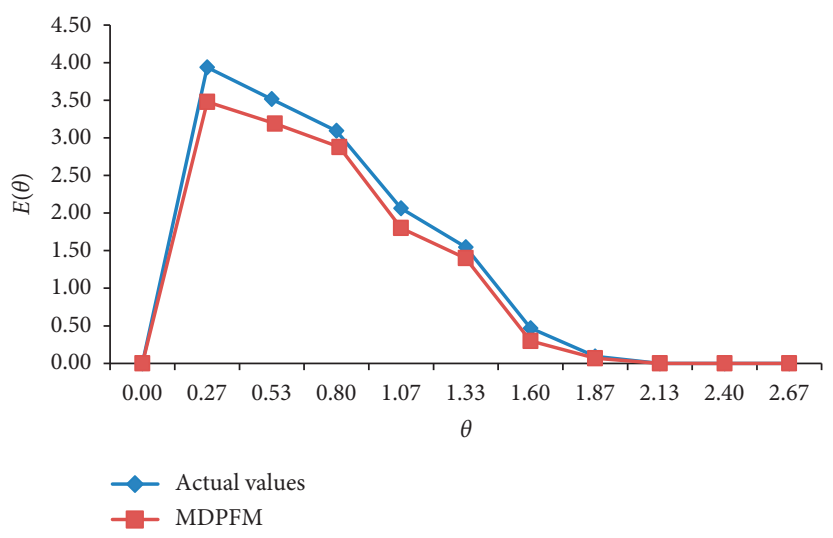

(d)

Figure 8: Prediction of $E(\theta)$ using MDPFM. (a) At $3.33 \times 10^{-5} \mathrm{~m}^{3} / \mathrm{sec}$. (b) At $6.67 \times 10^{-5} \mathrm{~m}^{3} / \mathrm{sec}$. (c) At $1 \times 10^{-4} \mathrm{~m}^{3} / \mathrm{sec}$. (d) At $1.33 \times 10^{-4} \mathrm{~m}^{3} / \mathrm{sec}$.

Equation (17) shows the complimentary influences of two important terms $e^{-0.5 \mathrm{Pe}(\theta+\theta 2 / 2)}$ and $\alpha q / Q C_{0}$. I term being the dispersion effects due to nonidealities in flow dynamics and II term shows the back flow effects owing to the liquid recirculation aided by gas evolution under electrolyzing mode. Understandably, from the above graphs, it is clear that nonidealities in flow currents get diminished by the back flow currents generated by gases which are inevitably produced at the electrode surface under electrolyzing conditions. From Figures 8(a) to 8(d), it is clear that MDPFM predicts RTD behavior of PBER under electrolyzing conditions.

\section{Conclusion}

RTD behavior of PBER was studied in various flow rates with and without electrolysis. Conventional dispersion models could explain the RTD behavior when PBER is operated without electrolysis. $D / u \cdot L$ values were determined by fitting in the dispersion model to assess the quantum of axial 
dispersion inside the packed bed. Modified Dispersion Plug Flow Model (MDPFM) was developed to predict the residence time distribution function $(E(\theta))$ under electrolyzing conditions. Finally, the model was validated in RTD studies using PBER operated under electrolyzing mode at various flow rates.

\section{Nomenclature}

$\begin{array}{ll}Q: & \text { Volumetric flow rate } \\ C_{0}: & \text { Initial concentration } \\ Q: & \text { Tracer quantity } \\ \alpha: & \text { Fraction of back flow } \\ C_{1}^{\prime}: & \text { Concentration immediately after the dispersed } \\ & \text { plug flow region } \\ C_{1}: & \text { Final output concentration } \\ T_{\mathrm{R}}: & \text { Tank residence time } \\ T: & \text { Time elapsed after injection of tracer } \\ \theta: & t / T_{\mathrm{R}} \\ \delta(t): & \text { Dirac delta function } \\ E(\theta) \text { or } E_{\theta}: & \text { Residence time distribution function } \\ D: & \text { Dispersion coefficient } \\ C: & \text { Concentration of species at any instance, } \\ & \text { u-velocity of electrolyte } \\ u: & \text { Velocity of electrolyte } \\ L: & \text { Length of bed } \\ Q: & \text { Bulk flow rate through the bed } \\ \text { Pe: } & \text { Peclet no. } u \cdot L / D \\ n_{\mathrm{T}}: & \text { No. of tanks in series } \\ \sigma^{2}: & \text { Variance } \\ \alpha_{\theta}^{2}: & \text { Variance (dimensionless) } \\ F_{\mathrm{T}}: & \text { Mass flow rate of tracer } \\ C_{\mathrm{T}}: & \text { Concentration of tracer } \\ U: & \text { Velocity. }\end{array}$

\section{Appendix}

\section{A. Dispersed Plug Flow Model Equation}

Exit age distribution $E_{\theta}$ as per dispersed plug flow model $=1 / \sqrt{\left(4 \pi \mathrm{Pe}^{-1}\right)} \times e^{-[(1-\theta) 2 \mathrm{Pe} / 4]}$.

Thus, as per the above model,

$$
\begin{aligned}
\frac{C_{1}^{\prime}(t)}{C_{0}} & =\frac{1}{\sqrt{\left(4 \pi \mathrm{Pe}^{-1}\right)}} \times e^{-\left[\left(1-t / T_{\mathrm{R}}^{2}\right) \mathrm{Pe} / 4\right]}, \\
C_{1}^{\prime}(t) & =\frac{C_{0}}{\sqrt{\left(4 \pi \mathrm{Pe}^{-1}\right)}} \times e^{-\left[\left(1-t / T_{\mathrm{R}}^{2}\right) \mathrm{Pe} / 4\right]} . \\
Q+\alpha Q & =Q(1+\alpha) .
\end{aligned}
$$

\section{A.1. Taking Material Balances}

At junction point $\mathrm{M}$,

$$
Q(1+\alpha) C_{1}^{\prime}=Q \cdot C_{1}+\alpha q \cdot \delta(t=0) .
$$

Taking Laplace transform across (A.3),

$$
Q \cdot C_{1}(s)+\alpha q=Q(1+\alpha) C_{1}^{\prime}(s) .
$$

We know,

$$
\begin{aligned}
C_{1}^{\prime}(s) & =\int_{0}^{\infty} e^{-s t} C_{1}(t) d t \\
& =\int_{0}^{\infty} e^{-s t} \times \frac{C_{0}}{\sqrt{\left(4 \pi \mathrm{Pe}^{-1}\right)}} \times e^{-\left[\left(1-t / T_{\mathrm{R}}^{2}\right) \mathrm{Pe} / 4\right]} d t \\
& =\int_{0}^{\infty} e^{-\mathrm{st}} \times \frac{C_{0}}{\sqrt{\left(4 \pi \mathrm{Pe}^{-1}\right)}} \times e^{-\left[-\mathrm{Pe} / 4 T_{\mathrm{R}}^{2}\left(t 2-2 t T_{\mathrm{R}}+T_{\mathrm{R}}^{2}\right)\right]} d t \\
& =\frac{C_{0}}{\sqrt{\left(4 \pi \mathrm{Pe}^{-1}\right)}} \times \int_{0}^{\infty} e^{-\left[\left(\mathrm{Pe} / 4 T_{\mathrm{R}} 2\right) t 2+\left(s+\mathrm{Pe} / 4 T_{\mathrm{R}}\right) t+(\mathrm{Pe} / 4)\right]} d t .
\end{aligned}
$$

From the standard form of integration results for exponential function,

$$
\int_{0}^{\infty} e^{-(a x 2+b x+c)} d x=\frac{1}{2} \times \sqrt{\left(\frac{\pi}{a}\right) e^{((b 2-4 a c) / 4 a)}} \times \operatorname{erfc}\left(\frac{b}{2 \sqrt{a}}\right),
$$

where $\operatorname{erfc}(p)=2 / \sqrt{\pi} \int_{0}^{\infty} e^{-x 2} d x, \quad a=\mathrm{Pe} / 4 \cdot T_{\mathrm{R}}^{2}, b=s+$ $\left(\mathrm{Pe} / 2 T_{\mathrm{R}}\right)$, and $c=\mathrm{Pe} / 4$.

Hence the solution of the integrand in (A.5) is

$$
\begin{aligned}
I_{1}= & \frac{1}{2} \sqrt{\frac{\pi}{\left(\mathrm{Pe} / 4 T_{\mathrm{R}}^{2}\right)}} \times e^{\left[\left(s+0.5 \mathrm{Pe} / T_{\mathrm{R}}\right) 2-\mathrm{Pe} / T_{\mathrm{R}}^{2} / \mathrm{Pe} / T_{\mathrm{R}}^{2}\right]} \\
& \times \operatorname{erfc}\left(\left(s+0.5 \mathrm{Pe} / T_{\mathrm{R}}\right) /\left(\frac{\sqrt{(\mathrm{Pe})}}{T_{\mathrm{R}}}\right)\right) .
\end{aligned}
$$

By simplifying,

$$
\begin{aligned}
I_{1}= & T_{\mathrm{R}} \sqrt{\left(\pi \mathrm{Pe}^{-1}\right)} \times e^{\left[(1 / \mathrm{Pe}) T_{\mathrm{R}}^{2}\left[s 2+s \cdot \mathrm{Pe} / T_{\mathrm{R}}\right]\right]} \\
& \times \operatorname{erf} c\left(\sqrt{\mathrm{Pe}^{-1}} \cdot T_{\mathrm{R}}\left(s+0.5 \mathrm{Pe} / T_{\mathrm{R}}\right)\right) .
\end{aligned}
$$

Thus from (A.5), $C_{1}^{\prime}(s)=C_{0} / \sqrt{\left(4 \pi \mathrm{Pe}^{-1}\right)} \times I_{1}$. From (A.4),

$$
\begin{aligned}
\mathbf{C}_{1}(\mathbf{s})= & (1+\alpha) \frac{C_{0}}{\sqrt{\left(4 \pi \mathrm{Pe}^{-1}\right)}} \times T_{\mathrm{R}} \sqrt{\pi \mathrm{Pe}^{-1}} \\
& \times \operatorname{erfc}\left(\sqrt{\mathrm{Pe}^{-1}} \cdot T_{\mathrm{R}}\left(\frac{s+0.5 \cdot \mathrm{Pe}}{T_{R}}\right)\right) \\
& \times e^{\left[(1 / \mathrm{Pe}) \cdot T_{\mathrm{R}}^{2}\left(s 2+s \cdot \mathrm{Pe} / T_{\mathrm{R}}\right)\right]}-\frac{\alpha q}{\mathrm{Q}} .
\end{aligned}
$$

\section{A.2. Finding $C_{1}(t)$ by Taking Inverse Laplace Transform of $C_{1}(s)$}

Compared with the standard form of results for inverse Laplace transform for product form of exponential and error function, 


$$
L^{-1}\left[e^{k 2 \cdot s 2} \times \operatorname{erf} c(k s)\right], \quad k>0=\frac{1}{(k \sqrt{\pi})} \cdot e^{(-(t 2 / 4 k 2))} .
$$

Value of complimentary error function in (A.9) is $n=$ $\sqrt{\mathrm{Pe}^{-1}} \cdot T_{\mathrm{R}}^{2} \cdot\left(s+0.5 \cdot \mathrm{Pe} / T_{\mathrm{R}}\right)$ and $n^{2}=\mathrm{Pe}^{-1} T_{\mathrm{R}}^{2}\left(s^{2}+s \cdot \mathrm{Pe} /\right.$ $\left.T_{\mathrm{R}}+\mathrm{Pe}^{2} / 4 T_{\mathrm{R}}^{2}\right)$.

If we modify the exponential term in (A.9) as $e^{(1 / \mathrm{Pe}) \cdot T_{\mathrm{R}}^{2} \cdot\left(s 2+\left(\mathrm{Pe} / T_{\mathrm{R}}\right) s+\mathrm{Pe} 2 / 4 T_{\mathrm{R}}^{2}\right)} \cdot e^{-\mathrm{Pe}}$, this term will be of the standard form $e^{k 2 \cdot s 2} \times \operatorname{erf} c(\mathrm{ks})$ having $k=\sqrt{\mathrm{Pe}^{-1}} \cdot T_{\mathrm{R}}$.

Thus, for a function, $F(s)=e^{k 2 \cdot\left(s+0.5 \mathrm{Pe} / T_{\mathrm{R}}\right) 2} \times \operatorname{erf} c(k$. $\left.\left(s+0.5 \mathrm{Pe} / \mathrm{T}_{\mathrm{R}}\right)\right), F(t)$ as per the standard solution mentioned above, $F(t)=1 /(k \cdot \sqrt{\pi}) \cdot e^{(-t 2 / 4 k 2)} \cdot e^{\left(-0.5 \mathrm{Pe} / T_{\mathrm{R}}\right) t} \cdot e^{-\mathrm{Pe}}$.

Thus,

$$
\begin{aligned}
C_{1}(t)= & (1+\alpha) \cdot \frac{C_{0}}{\sqrt{\left(4 \pi \mathrm{Pe}^{-1}\right)}} \cdot T_{\mathrm{R}} \cdot \sqrt{\left(\pi \cdot P e^{-1}\right)} \\
& \cdot \frac{1}{(k \cdot \sqrt{ } \pi)} \cdot e^{-\mathrm{Pe}} \cdot e^{(-t 2 /(4 k 2))} \cdot e^{\left(-\mathrm{Pe} / 2 T_{\mathrm{R}}\right) t}-\frac{\alpha q}{\mathrm{Q}} .
\end{aligned}
$$

Rearranging (A.11)

$$
C_{1}(t)=\left[0.5(1+\alpha) C_{0} \cdot \frac{T_{\mathrm{R}}}{k \sqrt{\pi}} \cdot e^{-\mathrm{Pe}}\right] \times e^{(-0.5 \mathrm{Pe}(\theta+\theta 2 / 2))}-\frac{\alpha q}{\mathrm{Q}} .
$$

Further simplifying and putting residence time distribution function as $E(\theta)=C_{1}(t) / C_{0}$,

$$
E(\theta)=(1+\alpha) \cdot \frac{e^{-\mathrm{Pe}}}{\left(2 \cdot \sqrt{\pi \mathrm{Pe}^{-1}}\right)} \times e^{(-0.5 \mathrm{Pe}(\theta+\theta 2 / 2))}-\frac{\alpha q}{\mathrm{QC}_{0}} .
$$

\section{Data Availability}

No data were used to support this study. All essential formulae are mentioned in Annexure.

\section{Conflicts of Interest}

The authors declare that they have no conflicts of interest.

\section{Acknowledgments}

The authors gratefully acknowledge the Vikram Sarabhai Space Research Centre, Trivandrum, and Cochin University of Science and Technology, Cochin, for supporting this work.

\section{References}

[1] S. H. Menon, A. M. Sadhik, M. Shaneeth, R. Raghu, J. Mathew, and G. Madhu, "Design and development of packed bed electrochemical reactors (PBER's) using scrap lead dioxide as novel electrodes," Journal of Chemical Engineering and Process Technology, vol. 6, no. 5, 2015.

[2] J. S. Newman and C. W. Tobias, "Theoretical analysis of current distribution in porous electrodes," Journal of the Electrochemical Society, vol. 109, no. 12, pp. 1183-1191, 1962.
[3] T. Doherty, J. G. Sunderland, E. P. L. Roberts, and D. J. Pickett, "An improved model of potential and current distribution within a flow-through porous electrode," Electrochimica Acta, vol. 41, no. 4, pp. 519-526, 1996.

[4] N. M. S. Kaminari, M. J. J. S. Ponte, H. A. Ponte, and A. C. Neto, "Study of the operational parameters involved in designing a particle bed reactor for the removal of lead from industrial wastewater-central composite design methodology," Chemical Engineering Journal, vol. 105, no. 3, pp. 111-115, 2005.

[5] G. Eigenberger and W. Ruppel, Catalytic Fixed-Bed Reactors, Wiley, New York, NY, USA, 2008.

[6] R. Saravanathamizhan, R. Paranthaman, N. Balasubramanian, and C. A. Basha, "Residence time distribution in continuous stirred tank electrochemical reactor," Chemical Engineering Journal, vol. 142, no. 2, pp. 209-216, 2008.

[7] T. Atmakidis and E. Y. Kenig, "Numerical analysis of residence time distribution in packed bed reactors with irregular particle arrangements," Chemical Product and Process Modeling, vol. 10, no. 1, pp. 17-26, 2015.

[8] O. Benhabiles, N. Chekir, and W. Taane, "Determining of the residence time distribution in CPC reactor type," Energy Procedia, vol. 18, pp. 368-376, 2012.

[9] A. D. Martin, "Interpretation of residence time distribution data," Chemical Engineering science, vol. 55, no. 23, pp. 5907-5917, 2000.

[10] O. Levenspiel, Chemical Reaction Engineering, Wiley, India, 3rd edition, 1999, ISBN: 978-81-265-1000-9.

[11] H. Scott Fogler, Elements of Chemical Reaction Engineering, Prentice Hall of India, India, 3rd edition, 2002, ISBN-81-2032234- 17. 


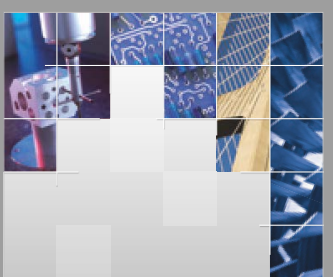

\section{Enfincering}
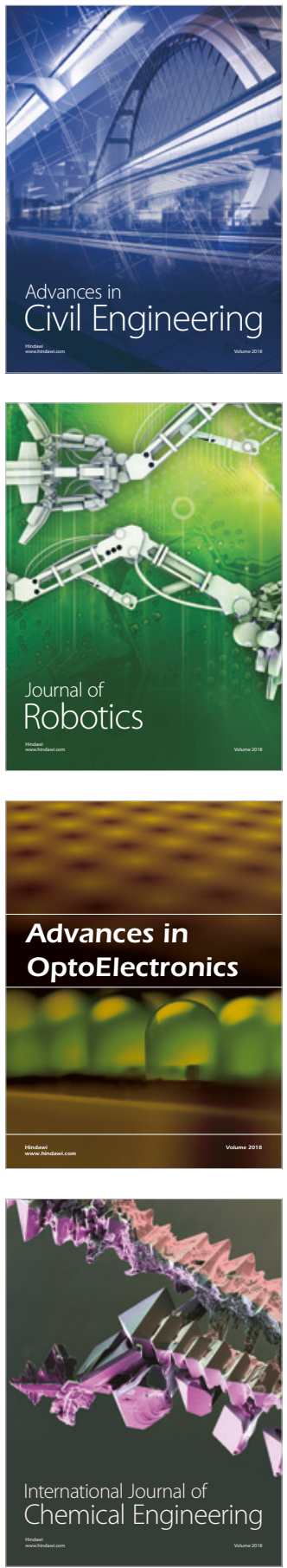

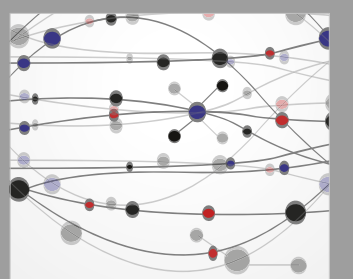

\section{Rotating \\ Machinery}

The Scientific World Journal

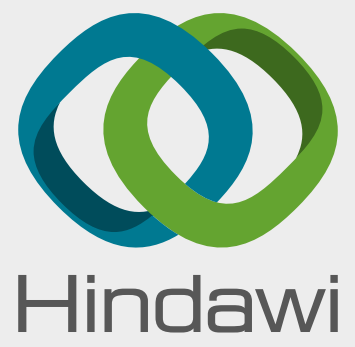

Submit your manuscripts at

www.hindawi.com
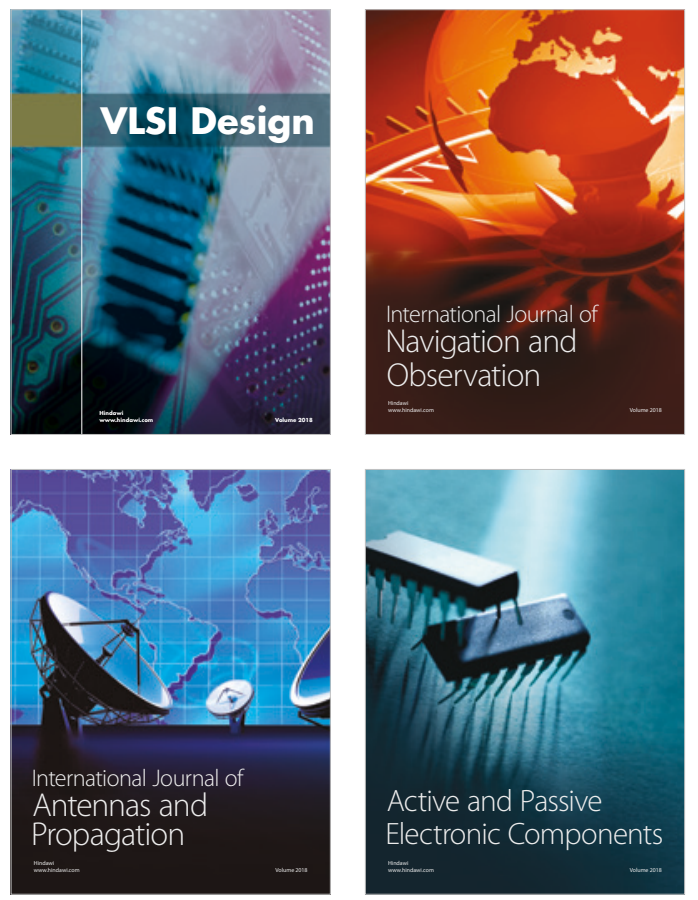
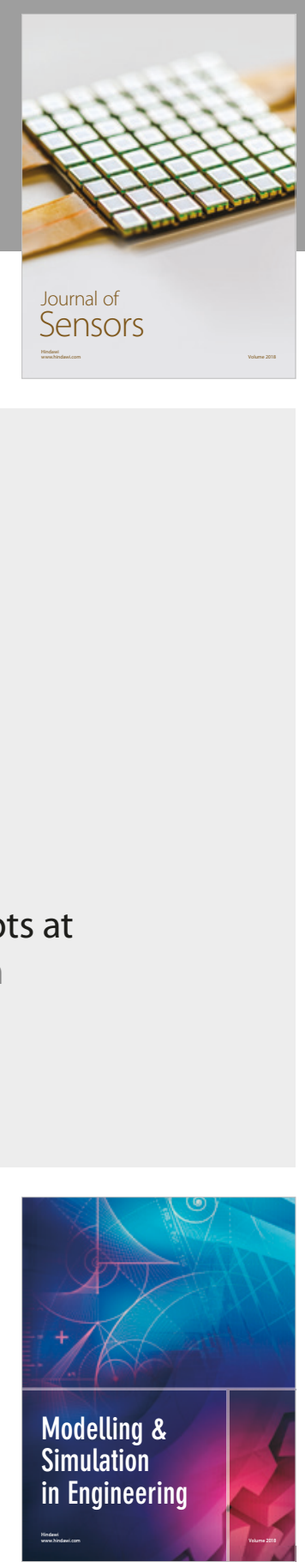

\section{Advances \\ Multimedia}
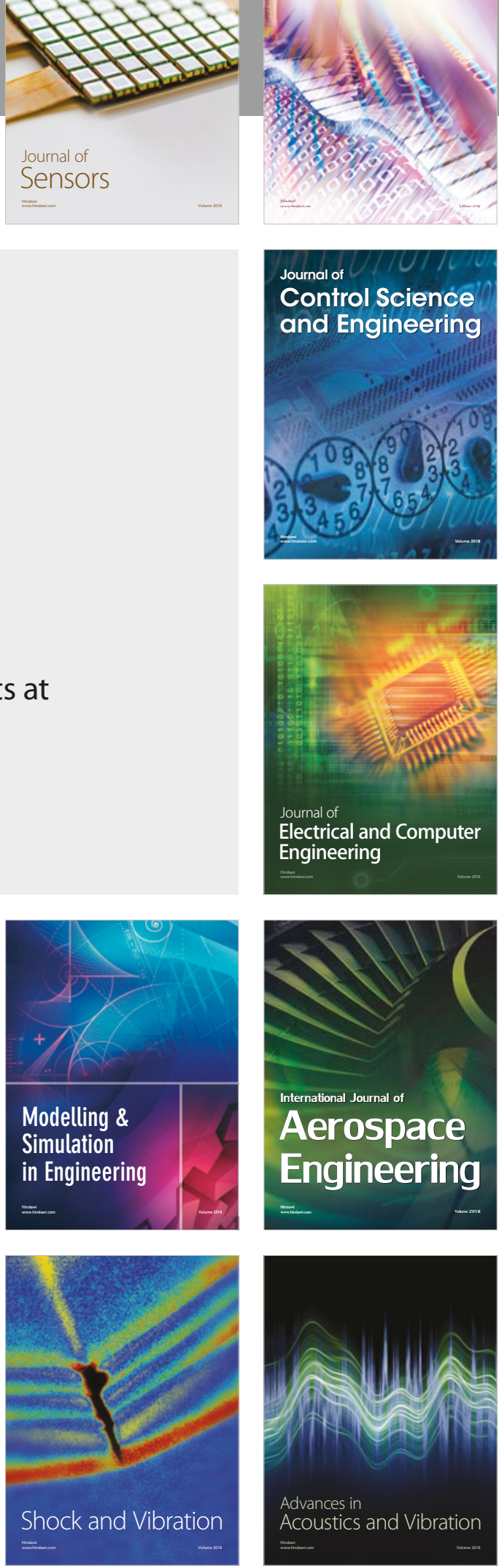